\title{
LA HOUILLE BLANCHE MET EN VALEUR UN DESERT
}

RICHMOND G. JOHNSON

Ingénieur au Bureau of Reclamation

Le «Grand Bassin silué principalement dans les Elats de Utah, Nevada of California, est un vaste deserl, partage par des chames de monlagnes nord-sud, en une centaine de euveltes sans issue vers la mer. Il y a peu de regions de cette taille aussi arides et de population aussi clairsemé, aux Elats-Unis. D'une surface de $518.000 \mathrm{~km}^{2}$, le bassin s'étend prineipalement entre les méridiens de $111^{\circ}$ et $121^{\prime \prime}$ de longitude onest et les parallèles $34^{\circ}$ et $44^{\prime \prime}$ de latitude nord. Bien que l'altitude générale du désert varie de 900 à 1.800 mèlres an-dessus du niveau de la mer, une des dépressions, "La Vallée de la Mort $\gg$ (Death Valley) est à 85 mètres audessous du niveau de la mer.

La plus grande partie des précipitations, dans ce bassin, tombent sur des pentes montagneuses où elles forment des rivières et des ruisseaux qui s'écoulent sur le sable du désert et y disparaissent ou se perdent dans les lacs qui s'assèchent lentement par évaporation, Le désert résulte d'un équilibre délicat entre la prépitation et l'évaporation qui a ste mantenu pendant des périodes immémoriales. Cependant, pendant une partie de l'ére plétoesne, l'équilibre fut détruit par des precipitations accues et une évaporation diminuée qui provoquirent l'accumulation d'eau dans deux cuvettes principales : les bassins du Lahontan et du Grand Lac Salé. Ce dernier bassin fut alor's rempli sur une hauteur de plus de 300 mètres et l'eau s'écoula dans le bassin de la rivière Columbia. Le rétablissement de l'équilibre provoqua l'abaissement des lacs par évaporation el, dans l'histoire de l'homme le Grand Lac Salé n'a jamais eu plus de 14 mètres de profondeur.

Les premiers colons pénétrèrent sur ce bassin il y a à peu près un siècle et, voyant la fertilité des anciennes terrasses lacustres, ils dérivèrent les rivières des montagnes et les ruisseaux pour cultiver les céréales et les chanvres. Ces premiers travaux d'irigation réussirent si bien que ces zones irriguces furent rapidement colonisées et furent développées d'une manière continue. Pendant la première décade, les surfaces cultivées ne dépassìrent pas les possibilités dirrigation offertes par l'étiage d'été, mais les colons qui vinrent ensuite furent obligés de se contente'r des quantités d'eau, quelquefois insuffisantes, qui leur restaient après satisfaction des droits d'eau des premiers occupants. Si grandes étaient les exigences alimentaires pout ces communautés en croissance que d'autres colons plus audacieux firent une tentative, relativement couronnée de succès, de cultures sans irrigation sur les pentes montagneuses qui recevaient un peu plus de précipitations que le plateau désertique. En laissant le sol en jachères pour accrottre l'humidité du sol, ces fermiers purent récolter jusqu'a 45 hl de blé par hectare, tous les deux ans.

La colonisalion el te développement finirent par mettre en valeur 350.000 hectares le long de la bordure orientale du bassin désertique et $\$ 00.000$ hectares dans ses parties septentrionales et occidentales. Neanmoins, la plupart de ces étendues n'avaient pas assez d'eau, particulierement pendant les années de faible pluie. Certaines annces, elles soufraient des crues. De hauts harrages dans les gorges furent nécessaires pour regulariser les rivières de montagne et pour accumuler les eaux d'hiver et les crues de prinlemps pour les besoins de l'agriculture. Ces travaux, effectués principalement pour la fournilure d'eau d'inrigation supplémentaire dépassaient les possibilités techniques et financières d'un petit groupe de fermiers; le Bureau of Reclamation du Gouvernement Fédéral des Etats-Unis aida les colons dans leurs projets, dans leurs études, leurs réalisations. En contrepartie, les fermiers acceptèrent de rembourser les frais au Gouvernement en quarante versements annuels. Il fut relativement facile aux 


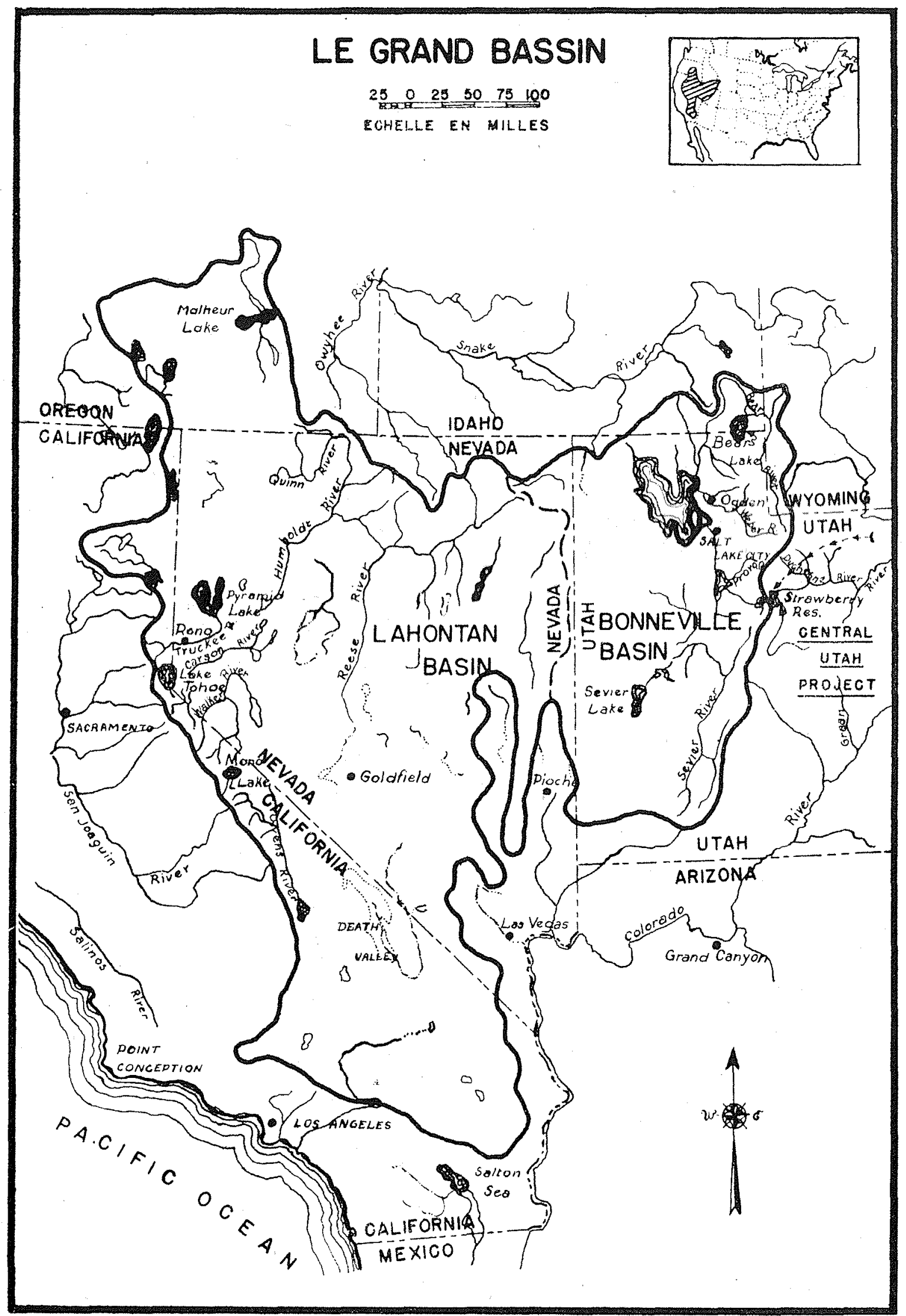

Figlure 1 


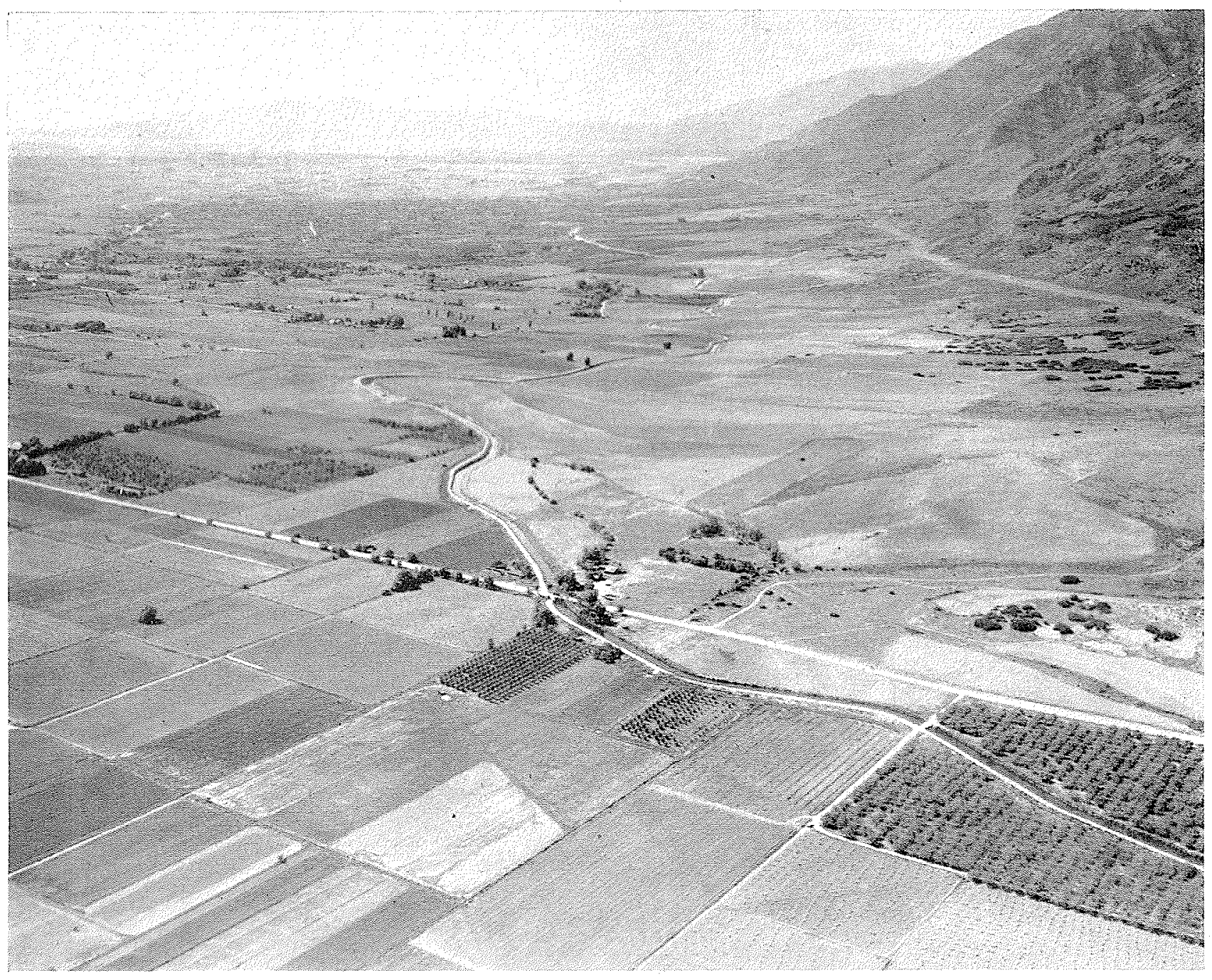

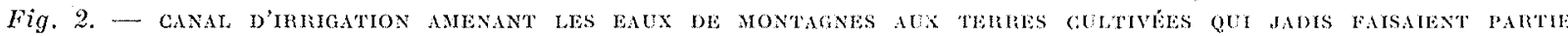
DU DÉSERT DU GRAND LAC SAIÉ,

Ingénieurs du Gouvernement d'édifier des ouvrages nécessaires à la fourniture d'eau d'irrigation supplémentaire pou'r 110.000 hectares de cultures mal irriguées et de l'eau pour plusieurs villes, moyennant la dépense totale de 30.000 .000 de dollars.

La tàche difficile est encore à fairre. Il reste encore 320.000 hectares qui ont désespérément besoin d'un surcroît d'eau, et le coût des travaux dépassera de beaucoup les possibilités de remboursement des cultivateurs. La solution financière se trouve dans des barrages à plusieurs buts, dont les revenus provenant de l'énergie hydroélectrique rembourseront la plus grande partie du prix de construction totale. En plus de l'eau d'irrigation supplémentaire, les travaux. proposés irrigueraient 180.000 hectares de nou- velles terres. La plupart des ouvrages faciliteraient aussi le contròle des crues et permettraient le développement des poissons et des animaux sauvages. Le prix des ouvrages projetés, s’ils ćtaient construits maintenant, est estimé à $1 / 2$ milliard de dollars.

Les trois plus grands projeis pour le « Great Basin 》 sont les ouvrages de Bear River, Central Utah, le long du coté oriental du bassin, et le projet de Lahontan, le long du côté occidental.

Le «Bear » (Rivière de l'Ours) est une rivière qui coule dans les Etats de Wyoming, Idaho èt Utah. Il y a un grand nombre d'années une compagnie privée construisit 5 centrales sur la rivière avec une puissance installée totale de $125.000 \mathrm{kw}$. A peu près $1 / 2$ milliard de mètres 
cubes d'eau s'écoulent annuellement par Ia centrale située à l'aval, passent dans le Grand Lac Salé et s'évaporent.

On a besoin de cette eau pou'r l'irrigation et une grande partie de l'eau disponible serait utilisée par le projet de Bear River pour irriguer 90.000 ha de nouvelles terres et pour compléter Ia fourniture d'eau insuffisante de 80.000 hectares de terres cultivées. Mais ces terres se trouvent à une altitude supérieure à celle des deux centrales aval de la rivière, de sorte qu'il est impossible d'utiliser l'eau, à la fois pour la fourniture d'énergie de ces centrales et pour l'irrigation. Done, pour rendre disponible cette eau pour l'irrigation, il faudrait installer: $60.000 \mathrm{kw}$ quelque part ailleurs, de facon à remplacer l'énergie fournie actuellement par ces deux centrales de la rivière. Ce remplacement pourrait ètre fait gràce aux grandes possibilités d'énergie du projet voisin de Central Utah.

Le plan d'équipement du projet Central Utah se dégagea après quarante-trois années d'études effectuées par des Ingénieurs, sur la possibilité de dériver à travers les montagnes et dans lo
Grand Bassin, une grande quantité d'eau prise dans le Green River (Rivière verte), affluent du Colorado. De nombreux projets de dérivation étaient pratiquement possibles, mais aucun n'était financièrement rentable avant la présentation du projet Central Utah. Essentiellement, ce projet dériverait l'eau du bras principal de la Rivière Verte à environ 1700 mètres d'altitude, pour l'irrigation de terres actuellement irrigućes par des affluents de la Rivière Verte.

Cette dérivation permettrait de rassembler les eaux des affluents de la Rivière Verte pour les faire traverser la montagne à haute altitude. Cette dérivation à haute altitude commencerait approximativement à 2440 mètres au-dessus du niveau de la mer et passerait à travers une série de 19 tunnels et de 14 conduites en béton jusqu'au Strawherry Reservoir à la cote de 2320 , ramassant en route l'eat de quelque vingt affluents. L'utilisation annuelle de 800.000 .000 de mètres cubes de ce réservoir passerail par un des tumnels relativement courts à travers la séparation Coloradomand Bassin et descendrait ainsi par 4 centrales ayant une chute totale de

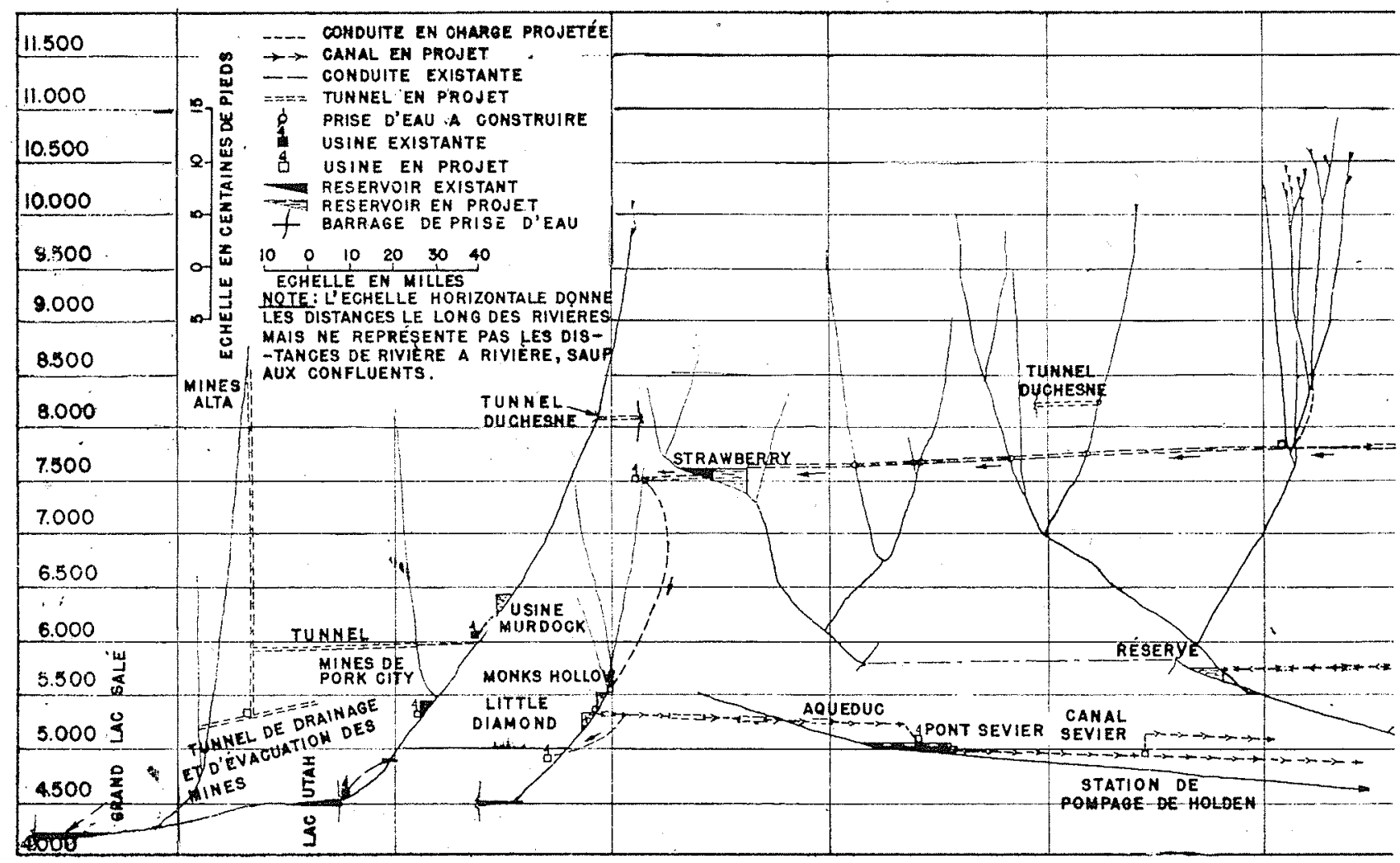


884 mètres avant d'atteindre le niveau du désert dans le Grand Bassin. A cet endroit, l'eau serait conduite par des canaux aux terres cultivables ayant besoin d'eat.

La longueur de l'aqueduc entre les limites extròmes du projet serait de plus de $480 \mathrm{~km}$ avec 26 tunnels d'une longueur totale de $175 \mathrm{~km}$. Le plus long tumnel aurait moins de $20 \mathrm{~km}$. de long.

Un grand ouvage de ce projet serait « Echo Park Dam "sur la Rivière Ve'te à une altilude qui élèverait le niveau de la rivière de 161, 2 metres; ce barrage aurait une capacité de 7,9 milliards de mètres cubes d'eau. La puissance installée de la centrale dépasserait $150.000 \mathrm{kw}$. Une des quatre centrales sur la pente du Gand Bassin et qui fonctionnerait sous une chute moyenne de 530 mètres aurait une capacité installice de $135.000 \mathrm{~km}$. Liensemble du projet comprendrait 9 centrales ayant une capacito totale installée de $410.000 \mathrm{kw}$ qui produirait plus de 1 milliard, 9 kwh par an.

Les problemes hydrauliques, rencontrés dans les études du projet Central Utah sont plus importants par leur échelle et par leur nombre que pour la plupart des projets de « Reclamation ». La source de l'eau est dans un pays tellement montagneux que 26 tunnels d'une longuetr de $175 \mathrm{~km}$ seratent nécessaires. Des conduites forcées auraient à faire descendre des centaines de millions de $\mathrm{m}^{3}$ d'eau chaque année, sur les pentes escarpées des montagnes. Des canaux devraient être étudiés pour amener de l'eau à des réservoirs pendant le climat t'rès dur de l'hiver. Le grand nombre de remplacements d'eau nécessaires pour échanger l'eau utilisée par les usagers actuels dans la Rivièe du Colorado pour l'amener aux usagers du Grand Bassin nécessiterait de nombreuses études hydrauliques. Ces études devront nécessairement s'étendre à l'exploitalion thérique hydraulique de 19 réservoirs dont 14 dependent les uns des autres de telle sorte que leur exploitation deva ctre simultanée. Les problemes hydrauliques sont compliqués en outre par les fonctions multiples des ouvages pour les usages agricoles, industriels, domestiques, le controle des crues, la propagation et la protection des poissons et du gibier d'cau, la récréation (natation et navigation) et le drainage des mines métalliques.

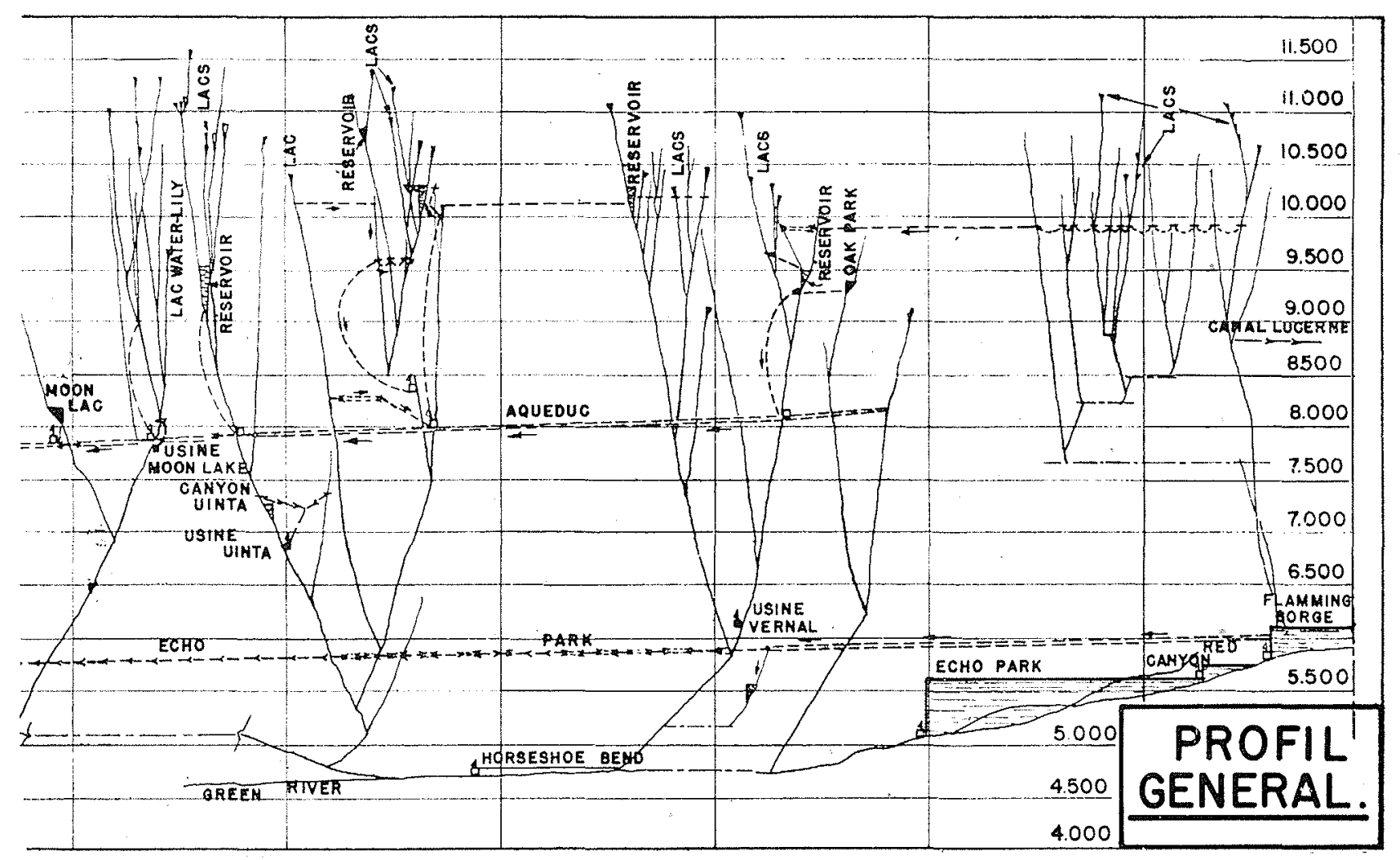

Figure 3 


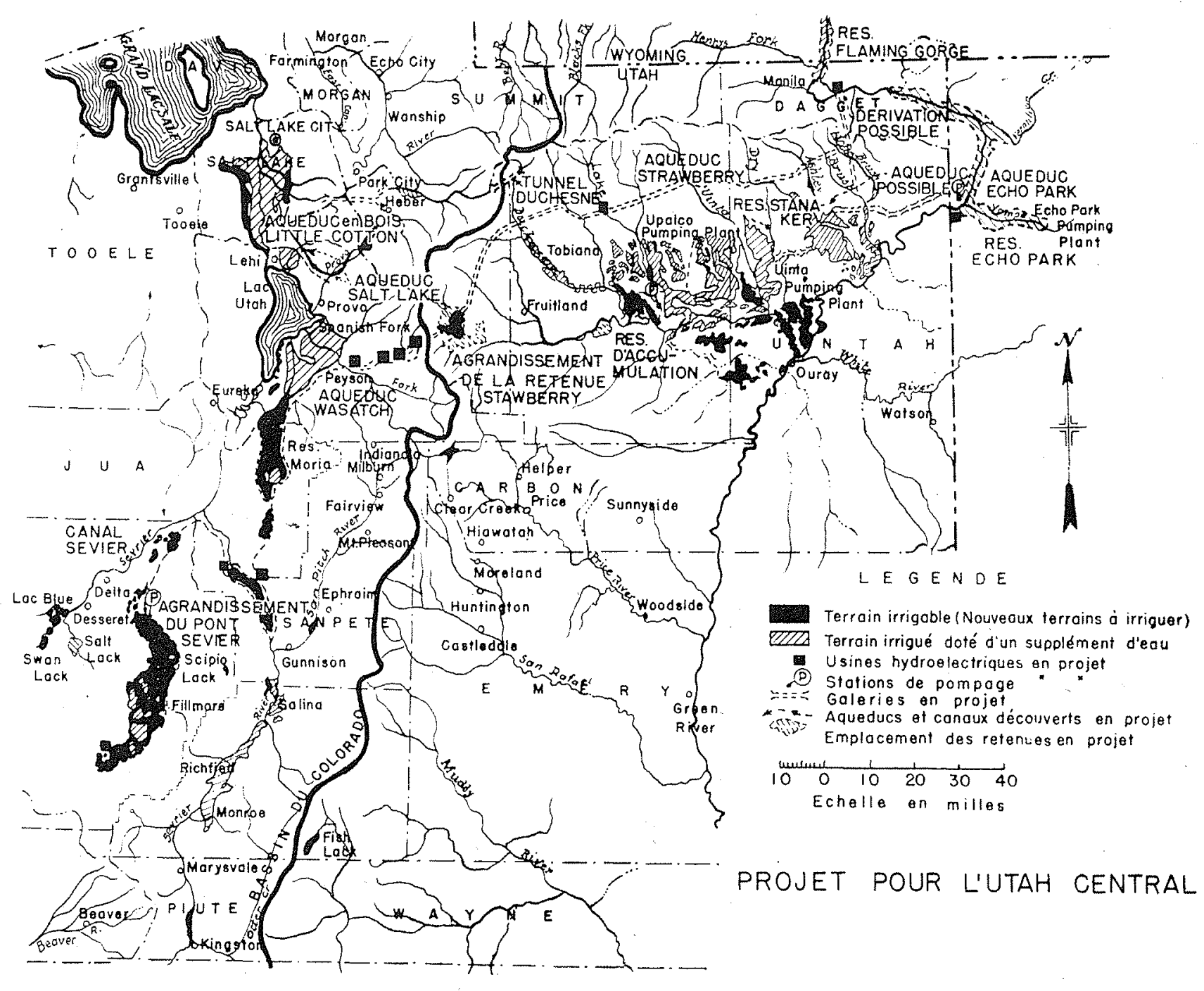

Figure \&

Le projet de Lahontan, sur la bordure occidentale du désert est intéressant. Il régulariserait trois rivières : La Truckee, la Carson et la Walker, issues de la Sierra Nevada et s'écoulant sur le plateau désertique, où il y a quelques terrains actuellement irrigués. Des crues dangereuses qui s'écoulent actuellement dans les trois petits résidus de l'ancien Lac Salé, pour s'y évaporer, seraient accumulées dans de hauts réservoirs pour la production de puissance et postérieurement pour l'irrigation de terres. L'ouvrage le plus important serait un réservoit à fonctions multiples situé su'r un affluent de la Rivière Carson à 2200 mètres au-dessus du niveau de la mer. L'eau serait enroyée dans ce réservoir depuis d'autres affluents par un système de tunnels collecteurs totalisant $45 \mathrm{~km}$. dont le plus long n'aurait que $8 \mathrm{~km}$. seulement. Les eaux prises dans le barrage s'écouleraient à travers deux centrales ayant une chute totale de 620 mètres. Ces centrales auraient une puissance installée de plus de $50.000 \mathrm{kw}$. et produiraient plus de 225 millions de kwh chaque anné. La puissance installée totale pour l'ensemble du projet des trois rivières serait de $135.000 \mathrm{kw}$ répartie entre 10 centrales avec une production amnuelle totale dépassant 600 millions de kwh annuellement.

Dans le Grand Bassin, des installations dont les projets considérés atteindraient une puissance totale de presque $550.000 \mathrm{kw}$ produiraient une énergie annuelle de 2 milliards, $5 \mathrm{kwh}$. La puissance produite par ces développements hydroélectriques envisagés, trouverait un marché disponible puisque le bassin avec ses vastes ressources minières importe actuellement d'énormes quantités d'énergie électrique. Les recettes provenant de l'énergie hydroélectrique 
paieraient, ultérieurement, la plus grande part du prix de construction des projets à destinations multiples, le solde des dépenses se trouvant largement dans la possibilité financière des fermiers à qui serait fournie de l'eau d'irrigation. Sans l'aide des recettes venant de l'énergie hydroćlectrique, ces terres ne pourraient pas être irriguées : La Houlle Blanche mettra en valeur un désert.

Même avec la réalisation de ces projets, des millions d'hectares de désert resteront sans eau, mais peut-être pas pour toujours. Au nord et à l'ouest du Grand Bassin, des rivières situées sur le versant côté Pacifique, comprenant la Columbia, contimueront à jeter chaque année $185 \mathrm{mil}$ - liards de mª d'eau dans l'Océan après que tous les besoins futurs sur ce versant auront été satisfaits. Ceci est un défi lancé aux Ingénieurs du Bureau of Reclamation. Ils savent déjà comment dériver plus de 5 milliards de $\mathrm{m}^{3}$ de cette eau, chaque année, dans le Grand Bassin, par un système de rétablissement de débit et de dérivation à travers les montagnes. Mais ceci ne serait qu'un petit aspect de ce plan d'utilisation des ressources d'eau occidentale à une échelle telle qu'il embrasserait et ferait bénéficier presque tous les projets de «Reclamation» de onze Etats de l'Ouest. Dix on vingt ans d'études séraient nécessaires mais, dès maintenant, on entrevoit de nouvelles perspectives pour la mise en valeur des déserts de l'ouest.

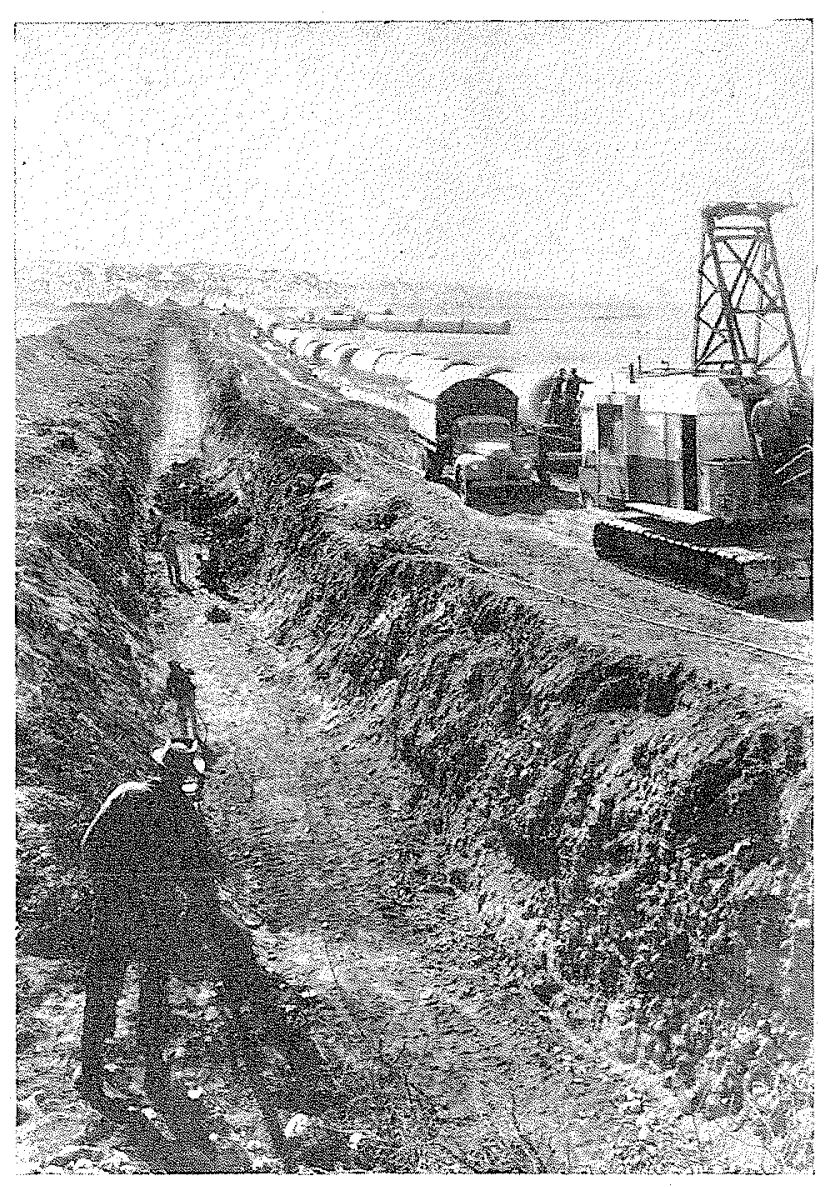

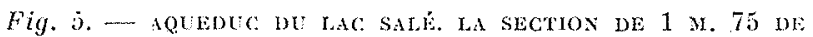
DMMITTR ET DF 64,4 KM. DE LONG ES'T MANTENaN" PRESQUE ACHEVĹE. 


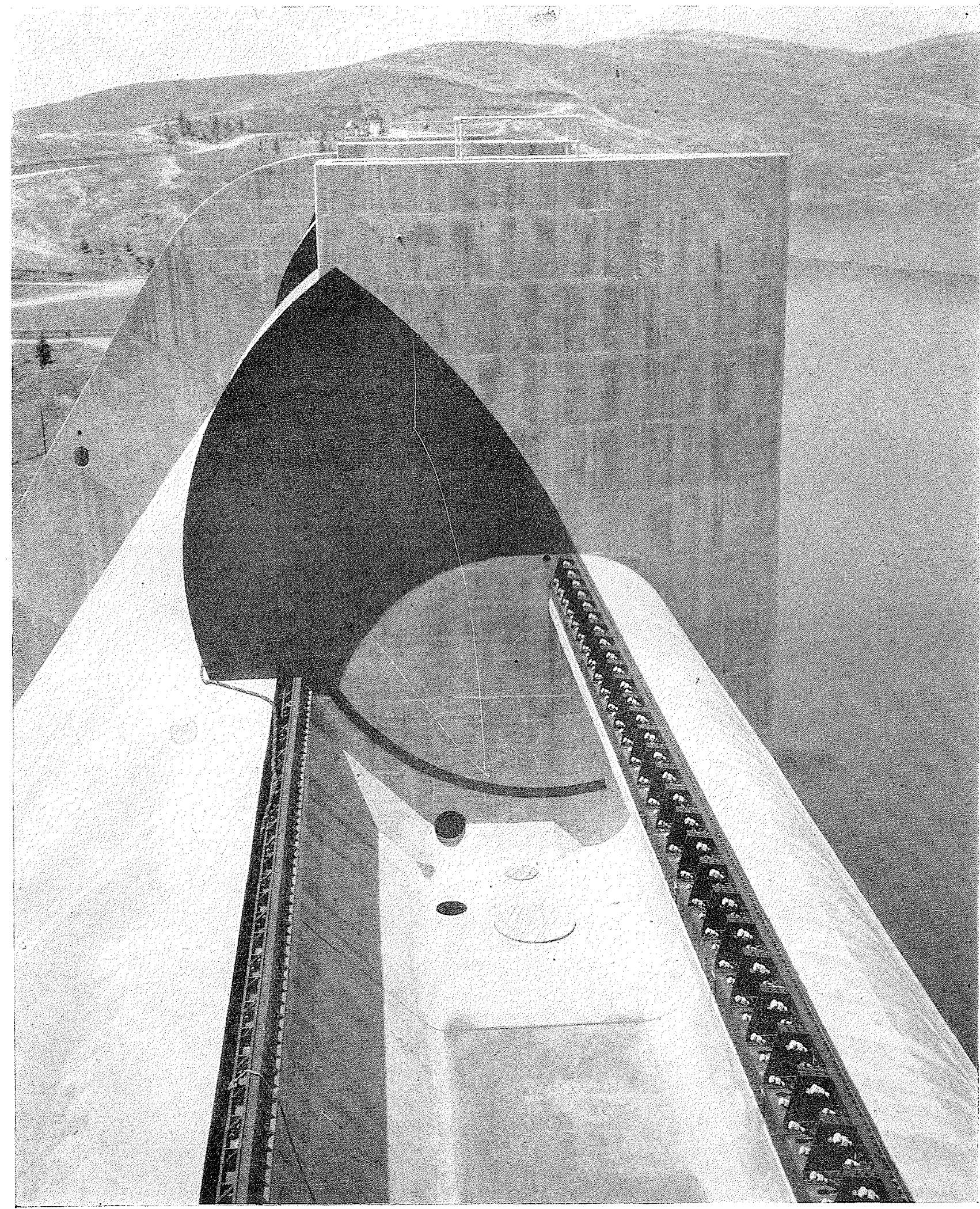

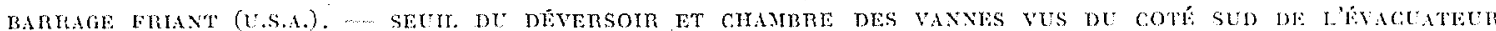

